\title{
FREE GROUPS IN LINDENMAYER SYSTEMS
}

\section{PETER JOHANSEN AND ERTK METLING}

Datalogisk institut, Københavns Universitet

Abstract. Locally catenative equations are defined in the free group. It is shown that if the free group generated by a DOL sequence is finitely generated then there exists a locally catenative equation in the free group which defines the DoI sequence. An algorithm is given which finds the generators of the free group if it is finitely generated. A conjecture is stated in terms of the existence of a certain group. The conjecture implies the solvability of the DOI equivalence problem.

\section{Introduction.}

Techniques from the theory of free groups are in this paper applied to the study of Iindenmayer systems. These techniques have previously been used by the authors in the study of regular languages 1,2. In the preliminaries follow the basic notation and results in free groups. No previous knowledge is assumed. In section 3 we consider locally catenative equations in the free group. See Rozenberg and Iindenmayer [95]. We define an equivalence group of two DOL systems and show that if there exists a finitely generated equivalence group then we can decide if the two systems are equivalent. Here we consider the sequence equivalence problem. See Mogens Nielsen [70] for a proof that this problem is equivalent to the language equivalence problem.

1. Peter Johansen, An Algebraic Normal Form for Regular Events, Polyteknisk Forlag, Lyngby 1972.

2. J.Clausen, J.Hammerum, E.Meiling, T.Skovgaard, Automata Theory in Free Groups, manuscript to be submitted to Aeta Informatica. 
We are not able to prove that a finitely generated equivalence group exists in the general case, nor that it can be effectively constructed. In special cases, however, it can be found. It is conjectured that it can be found in the general case. Section 4 presents an algorithm which finds the generators of the group closure of a DOL sequence if it is finitely generated.

We have, unfortunately, at present no way of deciding when this occurs. The algorithm is being implemented in IISP 1.5 on IBN/360.

\section{Preliminaries.}

For definitions and basic results in Lindenmayer systems and free groups the reader is referred to Herman and Rozenberg [45] and Hall $(1952)^{3}$.

In the following $H=\left\langle\Sigma, h, w_{0}\right\rangle$ and $G=\left\langle\Sigma, g, w_{0}\right\rangle$ will denote DOL systems. $h^{i}\left(w_{0}\right)$ will be denoted $w_{i}$ when reference to $H$ is implicit. The language generated by $\mathrm{H}$ is denoted $\mathrm{L}(\mathrm{H})=\left\{w_{0}, w_{1}, \ldots, w_{n}, \ldots\right\}$ The sequence generated by $H$ is denoted $E(H)=w_{0}, w_{1}, \ldots, w_{n}, \ldots$

The free group $F(\Sigma)$ is defined as follows: Let $\Sigma=\{a, b, \ldots, z\}$. Define a shadow alphabet $\Sigma=\{\bar{a}, \bar{b}, \ldots, \bar{z}\}$. Define an equivalence relation $D$ on the free semigroup $(\Sigma \cup \bar{\Sigma})^{*}$ as the transitive, symmetric, and reflexive closure of the adjacency relation $A$ :

$$
\begin{aligned}
& x A y \Leftrightarrow \exists u, v \in(\Sigma \cup \bar{\Sigma})^{*}, \exists c \in \Sigma \text { such that } \\
& x=u v \text { and }(y=u c \bar{c} v \text { or } y=u \bar{c} v)
\end{aligned}
$$

$F(\Sigma)$ is the set of equivalence classes of D. Group composition is $D(x) \cdot D(y)=D(x y)$. The t-element is the class $D(\lambda)$. The inverse of the class $D\left(a_{1}, a_{2} \ldots a_{n}\right)$ where $a_{i} \in(\Sigma \cup \bar{\Sigma})$ is the class $D\left(\bar{a}_{n} \ldots \bar{a}_{2} \bar{a}_{1}\right)$. Here $\overline{\bar{a}}_{i}$ denotes $a_{i}$.

3. M. Hall. The Theory of Groups, The Macmillan Company, New York, 1959. 
It can be shown that each equivalence class contains precisely one word which does not contain any adjacent occurrences of a letter and its inverse. This word is called a reduced word. An equivalence class is often denoted by its reduced representative.

Let $S \subset \mathbb{F}(\Sigma)$. [S] denotes the smallest subgroup of $\mathrm{F}(\Sigma)$ which contains S. S is called a generator set of [S]. [S] is called the group closure of S. Let $T \subset F(\Sigma)$ be a subgroup. If there exists a finite set $S$ such that $[s]=T$ then $T$ is finitaly generated. $y\left(s_{1}, s_{2}, \ldots, s_{n}\right)$ means an element of $\left[s_{1}, s_{2}, \ldots, s_{n}\right]$.

The main result on free groups is the following:

Let $A \subset F(\Sigma)$ be a group. There exists a set of generators $a_{1}, a_{2}, \ldots$, called free generators, such that $\left[a_{1}, a_{2}, \ldots\right]=A$ and such that any element from A can be written as a unique product of generators and their inverses. Any set with this property has the same cardinality, called the rank of $A$. The cardinality of any set of generators is at least the rank of $A .\left\{a_{i}\right\}$ can furthemore be chosen such that $a_{i}=u_{i} s_{i} \bar{v}_{i}, i=1,2 \ldots$ where no letters from $s_{i}$ or $s_{j}$ are cancelled in any products $a_{i} \pm 1 a_{j} \pm 1$. s $s_{i}$ is called the significant factors of $a_{i}$.

Example 1:

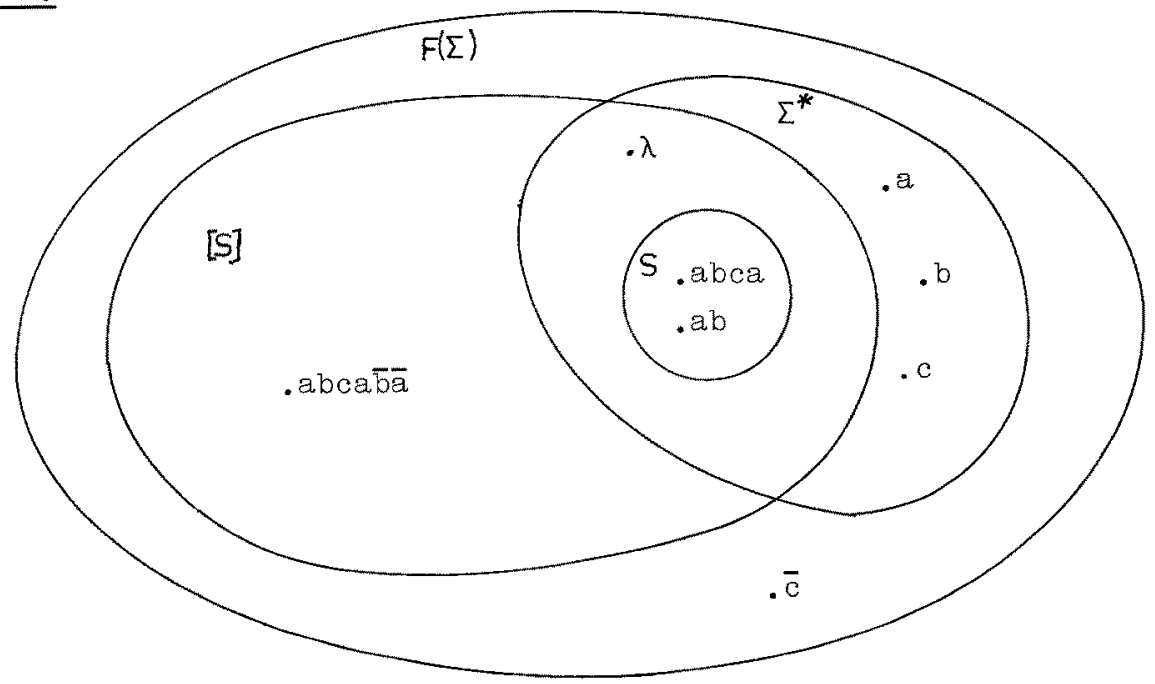


Example 2:

$$
A=\underset{\wedge}{[a b \bar{a},} a c]=\underset{\wedge}{[a b c}, a c]=\underset{M}{[a b c a c}, a c]
$$

Significant factors are marked by $\wedge^{*}$

Example 3:

$$
\begin{aligned}
& F(\Sigma)=\underset{\wedge}{[a}, b] S=[a a, a b \bar{a}, b] \\
& T=\left[a a b, a a a a b b, \ldots,(a a)^{2^{n}} b^{2^{n}}, \ldots .\right. \\
& T \subset S \subset F(\Sigma) .
\end{aligned}
$$

This example shows that the rank of a subgroup may be greater than the rank of the group itself:

$\operatorname{rank}(F(\Sigma))=2 ; \operatorname{rank}(S)=3 ; \operatorname{rank}(T)=\infty$.

That rank $(T)=\infty$ can be seen this way:

Let $s_{n}=(a a)^{2^{n}} b^{2^{n}} \cdot T=\left[s_{0}, s_{1}, \ldots\right]$

Define $t_{n}=s_{n} \bar{s}_{n-1}$ for $n=1,2, \ldots$ and $t_{0}=s_{0}$.

This gives $t_{n}=(a a)^{2^{n}} b^{2^{n}} \bar{b}^{2^{n-1}}(\bar{a} \bar{a})^{2^{n-1}}$

$$
=(a \mathrm{a})^{2^{n}} b^{2^{n-1}}(\bar{a} \bar{a})^{2^{n-1}}
$$

It is seen that $T=\left[t_{0}, t_{1}, \ldots\right]$ because $s_{n}=t_{n} t_{n-1} t_{n-2} \ldots t_{0}$. Also it is seen that the occurrences of $b$ in $t_{n}$ constitute significant factors. Hence $\operatorname{rank}(T)=\infty$.

\section{Locally catenative equations in the Free Group:}

First the case is studied where $[\mathrm{I}(H)]$ is finitely generated. An equivalence group of two DOL-systems is defined. Theorem 2 shows that if a finitely generated equivalence group can be found for two systems, then the equivalence problem is solved. Then it is conjectured that a finitely generated equivalence group always exists, and can effectively be 
constructed.

Theorem 1:

$[\mathrm{L}(\mathrm{H})]$ is finitely generated

There exists a locally catenative equation in $F(\Sigma)$ :

$$
w_{n}=e q\left(w_{n-1}, \ldots, w_{n-r}\right) \text { for } n \geq \text { cut }
$$

\section{Proof:}

$\Downarrow:$ Let $[I(H)]=\left[g_{1}, \ldots, g_{n}\right]$. Each $g_{k}, k=1, \ldots, n$, is a product $g_{k}\left(w_{0}, w_{1}, \ldots, w_{k^{-1}}\right)$ for some $q_{k_{k}}$. Let $r$ be the maximum of all $q_{k}$. Then $[I(H)]=\left[w_{0}, w_{1}, \ldots, w_{r-1}\right]$. From this it follows that $w_{r} \in\left[w_{0}, w_{1}, \ldots, w_{r-1}\right]$ and $w_{r}=e q\left(w_{r-1}, \ldots, w_{0}\right)$ since $h$ is a homomorphism of $\mathbb{E}(\Sigma)$ this part follows by induction.

A: From the recursion equation it follows by induction that $[\mathrm{H}(\mathrm{H})]$ is generated by $\left\{w_{0}, w_{1}, \ldots, w_{r-1}\right\}$.

At this point a natural question arises:

If the rank of $[\mathrm{L}(\mathrm{H})]$ is $r$, can we thenchoose the first $r$ elements from $\mathrm{E}$ (H) as generators? We have been unable to prove this, but believe it to be true.

\section{Small conjecture.}

$[\mathrm{H}(\mathrm{H})]$ is finitely generated of rank $r$<smiles>C1=C2C=C12</smiles>

$[L(H)]$ is freely generated by $\left\{w_{0}, w_{1}, \ldots, w_{r-1}\right\}$

This conjecture is mentioned again in section 4 in connection with the algorithm to find free generators of $\mathrm{L}(\mathrm{H})$. Here we shall point out a consequence of this small conjecture, which follows immediately from the unique factorization in the free group. 


\section{Consequence of small conjecture.}

$[I(H)]$ is finitely generated of rank $r$

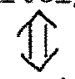

There exists a unique locally catenative equation in the free groups of minimal cut with cut $=r$ and depth $\leq r$.

\section{Example 4:}

$$
\begin{aligned}
E(H) & =a b, a b a b, \ldots \\
E(H) & =[a b] \text { of rank } 1 \\
& w_{1}=a b a b=w_{0} w_{0} \\
& \text { recurrence equation } \\
& w_{n}=w_{n-1} w_{n-1}
\end{aligned}
$$

\section{Example 5:}

$$
\begin{aligned}
& H=\langle\{a, b\},\{a \rightarrow a, b \rightarrow a b\}, b\rangle \\
& E(H)=b, a b, a a b, \ldots \\
& G=\langle\{a, b\},\{a>a, b \rightarrow b a\}, b\rangle \\
& E(G)=b, b a, \text { baa, } \ldots \\
& \quad[I(H)]=[I(G)]=[a, b] \text { of rank } 2
\end{aligned}
$$$$
\text { recurrence equation in common: }
$$$$
w_{n}=w_{n-1} \bar{w}_{n-2} w_{n-1}, \text { cut }=2
$$$$
E(H) \text { defined by } w_{0}=b, w_{1}=a b
$$$$
E(G) \text { defined by } w_{0}=b, w_{1}=b a \text {. }
$$

\section{Example 6:}

$$
\begin{aligned}
G=\langle\{a, b, c\},\{a \rightarrow c b a, b>a, c \rightarrow c c\}, b\rangle \\
w_{0}=b \\
w_{1}=a \\
w_{2}=c b a
\end{aligned}
$$

Since $a=w_{1}, b=w_{0}$, and $c=w_{2} \bar{w}_{1} \bar{w}_{0}$ we know that $[\mathrm{I}(H)] \supset F(\Sigma)$ and 
therefore $[\mathrm{L}(\mathrm{H})]=F(\Sigma)$.

$$
w_{3}=\text { ccacba }
$$

It then follows that $w_{3}$ can be expressed in terms of $w_{0}, w_{1}$, and $w_{2}$ by substitution:

$$
\begin{aligned}
w_{3} & =\left(w_{2} \bar{w}_{1} \bar{w}_{0}\right)\left(w_{2} \bar{w}_{1} \bar{w}_{0}\right) w_{1}\left(w_{2} \bar{w}_{1} \bar{w}_{0}\right) w_{0} w_{1} \\
& =\left(w_{2} \bar{w}_{1} w_{0}\right)^{2} w_{1} w_{2}
\end{aligned}
$$

The recurrence equation becomes

$$
w_{n}=\left(w_{n-1} \bar{w}_{n-2} \bar{w}_{n-3}\right)^{2} w_{n-2^{2}} w_{n-1}
$$

\section{Example 7:}

$$
H=\langle\{a, b\},\{a \rightarrow a a, b \rightarrow b b\}, a a b\rangle
$$

$[\mathrm{H}(\mathrm{H})]$ is of infinite rank as shown in example 3. By theorem 1 it follows that there does not exist a locally catenative recurrence equation defining $\mathrm{E}(\mathrm{H})$. Exarnple finished.

We shall in what follows attempt to formulate a generalization of theorem 1. We have to present it as a conjecture since we are unable to prove it.

Definition: An equivalence group for the two DOL systems $H$ and $G$ is a group $Q$ with the properties

$$
\begin{array}{ll}
\text { (i) } & w_{0} \in Q \\
\text { (ii) } & h(Q) \subset Q \text { and } g(Q) \subset Q \\
\text { (iii) } & (\forall q \in Q)\left[(\forall i) h^{i}\left(w_{0}\right)=g^{i}\left(w_{0}\right)\right] \Rightarrow\left[(\forall i) h^{i}(q)=g^{i}(q)\right]
\end{array}
$$

\section{Lemma 1:}

For any DOI systems such that $[I(H)]=[I(G)],[I(H)]$ is an equivalence group. 


\section{Proof:}

(i) and (ii) are trivially fulfilled. To verify (iii), assume that $H$ and G are equivalent.

Let $q \in[H(H)] \cdot h^{\dot{i}}(q)=h^{i}\left(q\left(w_{0}, h\left(w_{0}\right), \ldots, h^{j}\left(w_{0}\right), \ldots\right)\right)$

$$
=q\left(h^{i}\left(w_{0}\right), h^{i+1}\left(w_{0}\right), \ldots\right)
$$

Since $h^{j}\left(w_{0}\right)=g^{j}\left(w_{0}\right)$ for all $j$, we get

$=q\left(g^{i}\left(w_{0}\right), g^{i+1}\left(w_{0}\right), \ldots\right)$

$=g^{i}\left(q\left(w_{0}, g\left(w_{0}\right), \ldots\right)\right)$

$=g^{i}\left(q\left(w_{0}, h\left(w_{0}\right), \ldots\right)\right)=g^{i}(q)$

\section{Iemma 2:}

There exists only one DOL-system $\mathrm{H}$ which generates $\mathrm{E}(\mathrm{H})$. $F(\Sigma)$ is an equivalence group for all DoI systems $G$.

\section{Proof:}

U: Obvious because (iii) is vacuously fulfilled.

$\mathbb{1}$ : Obvious because (iii) implies that $g$ and $h$ are identical on the generators of $F(\Sigma)$.

\section{Example 8:}

The DOL-system from example 7 is uniquely determined by $E(H)$. Hence $[a, b]$ is an equivalence group for all DOL-systems $G$.

The significance of equivalence groups is due to the next theorem.

\section{Theorem 2:}

If there exists a finitely generated equivalence group $Q=\left[q_{1}, \ldots, q_{n}\right]$ for two DoL-systems $G$ and $H$ then $G$ and $H$ are equivalent if and only if

$$
\text { (*) } h\left(q_{i}\right):=g\left(q_{i}\right) \text { for } i=1,2, \ldots, n
$$

\section{Proof:}

Assume $G$ and $H$ equivalent; then (iii) implies the theorem.

Assume (*). We are going to prove $\forall i \geq 0\left[h^{i}\left(w_{0}\right)=g^{i}\left(w_{0}\right)\right] .(*)$ implies, that $\forall q \in Q \quad[h(q)=g(q)]$

Since $Q$ is closed under the homomorphisms $G$ and $H$ we conclude that 
$\forall i \geq 0 \forall q \in Q \quad\left[h^{i}(q)=g^{i}(q)\right]$

The result follows now from the fact that $w_{0} \in Q$.

\section{Coroliary 1:}

If $[L(H)]=[L(G)]=\left[w_{0}, h\left(w_{0}\right), \ldots, h^{r-1}\left(w_{0}\right)\right]$

then $G$ and $H$ are equivalent if and only if

$$
h^{i}\left(w_{0}\right)=g^{\dot{I}}\left(w_{0}\right) i=0,1, \ldots, r
$$

\section{Proof:}

Follows from lemma 1 and theorem 2.

\section{Example 9:}

Using corollary 1 we can, with reference to example 6, deduce that two DOI-sequences which have the common initial terms

$$
\begin{aligned}
& \mathrm{w}_{0}=\mathrm{b} \\
& \mathrm{w}_{1}=\mathrm{a} \\
& \mathrm{w}_{2}=\text { cba } \\
& \mathrm{w}_{3}=\text { ccacba }
\end{aligned}
$$

must be identical.

Example 10:

$\mathrm{H}=\langle\{a, b, c, d\},\{a \rightarrow a a, b \rightarrow b, c \rightarrow c, d \rightarrow b d\}, a c d\rangle$

$G=\langle\{a, b, c, d\},\{a \rightarrow a a, b \rightarrow b, c \rightarrow c b, a \rightarrow d\}, a c d\rangle$

$\mathrm{E}(G)=\mathrm{E}(H)=\operatorname{acd}$, aacbd, aaaacbbd, $\ldots,(a a)^{2^{n}} \mathrm{cb}^{\mathrm{n}} \mathrm{d}, \ldots$

We notice that the occurrences of c constitute significant factors. Hence the rank of $[\mathrm{I}(\mathrm{H})]$ is infinite and the equivalence group $[\mathrm{L}(\mathrm{H})]$ is infinitely generated. Lemma 2 tells us that $[a, b, c, d]$ is not an equivalence group.

All powers of $h$ and $g$ coincide on $a$ and on $b$, and

$$
h^{i}(c d)=g^{i}(c d)=c b^{i} d \text { for } i=0,1, \ldots
$$

From this follows that $\mathrm{T}=\left[\mathrm{a}, \mathrm{b}, \mathrm{cd}, \mathrm{cbd}, \mathrm{cb}^{\dot{i}} \mathrm{~d}, \ldots\right]$ is an equivalence 
group. .

$$
T=\underset{\wedge}{[a}, \underset{\wedge}{b}, \underset{\wedge}{c d}] \text { since } \mathrm{cb}^{\dot{i}} \mathrm{~d}=(\mathrm{cbc})^{\dot{1}} \mathrm{~cd}
$$

We have found a finitely generated equivalence group for $G$ and $H$ even if $[L(G)]=[\amalg(H)]$ is infinitely generated.

We proceed to formulate a conjecture.

\section{Main conjecture:}

For any two DOL-systems $H$ and $G$ a finitely generated equivalence group can be effectively constructed.

Because of theorem 2, a. proof of this conjecture will solve the equiva. lence problem. for DOL-systems.

A consequence of the conjecture is that for any DOI sequence $H$ the set of all free group elements $x$, with the property that if $G$ is equivalent with $H$ then $\langle\Sigma, h, x\rangle$ is equivalent with $\langle\Sigma, g, x$, , is a finitely generated group. This follows because the intersection of two finitely generated subgroups of $F(\Sigma)$ is finitely generated, and because there exists only a finite number of DOL systems equivalent to $H$. (M. Nielsen $[70]$ ).

4. An algorithm to find $[\mathrm{L}(\mathrm{H})]$.

This section presents an algorithm which finds a set of generators for [L(H)] if this group is finitely generated. If this is not so, the algorithm yields a still larger subset of an infinite set of free generators with significant factors. We have at present no available test to decide between the two possibilities. It is no surprise that this algorithm exists. Nielsen's algorithm is known from free group theory. It finds a set of free generators with significant factors from a finite set of generators.

Let Nielsen (W) be a procedure which has a finite set W as argument and which as result delivers a set of free generators with significant factors of $[\mathrm{W}]$. 
Our algorithm is then as follows:

begin

$Y_{0}:=\left\{w_{0}\right\} ;$

$\mathrm{n} \quad:=1$;

while $w_{n} \notin\left[Y_{n-1}\right]$ do

begin $Y_{n}:=\operatorname{Nielsen}\left(Y_{n-1} \cup\left\{w_{n}\right\}\right)$

n $:=n+1$

end

end

At exit from the algorithm, $w_{n} \in\left[Y_{n-1}\right]$. It follows that $\left[I_{(H)}\right]=\left[Y_{n-1}\right]$. It is believed, and stated as the small conjecture in section 3 , that the rank of $[\mathrm{L}(\mathrm{H})]$ equals $n$ at exit from the algorithm. The sore point is of course that if $[\mathrm{L}(\mathrm{H})]$ has infinite rank then the algorithm never exits. In this case $[I(H)]=\bigcup_{i=0}^{\infty}\left[Y_{i}\right]$. An adaptation of the algorithm, which takes into account that the sequence is generated by a nomomorphism is being implemented in IISP 1.5 on IBM/360. In the next examples, $\mathrm{Z}_{\mathrm{n}}$ denotes $\left[\mathrm{Y}_{\mathrm{n}}\right]$.

\section{Example 11:}

This example shows the successive values of $z_{n}$ for the following DOIsystem

$$
H=\langle\{a, b, c\},\{a-\rangle a b, b-\rangle c a, c-\rangle a c\}, a c\rangle
$$

The first elements of the sequence are 
$z_{0}=[x]$

$z_{1}=[a, c, a b a c]=[a c, a b]$

$z_{2}=[a c, a b, a b c a b a c]=[a c, a b, c a]$

$z_{3}=[a c, a b, c a, a b c a a c a b a b c a a b a c]=\left[a_{x}, a c_{x}, c_{x}, \lambda\right]=z_{2}$

Now the chain $Z_{0} \subset Z_{1} \subset Z_{2} \ldots$ will stay constant forever and $\left[I_{r}(H)\right]=Z_{2}$. From theorem 1 we know that the sequence is locally catenative. From lemma 1 we know that $[\mathrm{L}(\mathrm{H})]$ is an equivalence group for any other DOLsystem that generates the same sequence.

From an algorithmic point of view it would be convenient to find generators for $Z_{n}$ as an extension of the generators found for $Z_{n-1}$, and this is always possible since $z_{n}$ is an extension of $z_{n-1}$. In this example it is even possible to obtain significant factors in the extended set of generators. This is not always possible as will be shown in example 12.

We know that the DOL-system $\mathrm{H}$ is locally catenative but we have not found a locally catenative formula. Such a formula can be found by keeping track of the relation between the generators of $z_{n}$ (in the following denoted $w_{0}^{\prime}, \ldots, w_{n-1}^{\prime}$ ) and the elements of the sequence $\left(w_{0}, \ldots, w_{n}, \ldots\right)$. We will also use the fact that

$$
z_{n}=\left[w_{0}^{\prime}, \ldots, w_{n-1}^{\prime}, h\left(w_{n-1}\right)\right]
$$

Using this method we get

step 0

$\mathrm{w}_{\mathrm{o}}=\mathrm{ac}$

$$
w_{0}^{1}=w_{0}
$$

$z_{0}=\left[w_{0}^{i}\right]=[\mathrm{d} g]$

step 1

$h\left(w_{0}^{1}\right)=$ abac

$w_{1}^{1}=h\left(w_{0}^{1}\right) \bar{w}_{0}^{t}=a b$

$w_{1}^{\prime}=w_{1} \bar{w}_{0}$

$z_{1}=\left[w_{0}^{*}, w_{\dagger}^{\dagger}\right]=\left[a c_{1}, a b_{0}\right]$ 


\section{step 2}

$h\left(w_{1}^{\prime}\right)=a b c a$

$w_{2}^{\prime}=\bar{w}_{1}^{t} h\left(w_{1}^{\prime}\right)=c a$

$z_{2}=\left[w_{0}^{1}, w_{1}^{1}, w_{2}^{1}\right]=\left[a_{1}, a_{\lambda}, c_{x}\right]$

$$
w_{2}^{\prime}=w_{0} \bar{w}_{1} \cdot w_{2} \bar{w}_{1}
$$

step 3

$\mathrm{h}(\mathrm{w} !)=\mathrm{acab}$

$\mathrm{w}_{3}^{\prime}=\bar{w}_{1}^{\prime} \bar{w}_{0}^{\prime} h\left(\mathrm{w}_{2}^{*}\right)=\lambda$

$z_{3}=\left[w_{0}^{1}, w_{1}^{1}, w_{2}^{1}, w_{3}^{1}\right]=\left[a_{x}, a x_{1}, c_{c}^{a}\right]$

$\mathrm{w}_{3}^{\prime}=\mathrm{w}_{0} \overline{\mathrm{w}}_{1} \cdot \overline{\mathrm{w}}_{0} \cdot \mathrm{w}_{1} \overline{\mathrm{w}}_{2} \mathrm{w}_{3} \overline{\mathrm{w}}_{2}$

From the calculations in step 3 we get

$$
\begin{aligned}
& \lambda=w_{0} \bar{w}_{1} \bar{w}_{0} w_{1} \bar{w}_{2} w_{3} \bar{w}_{2} \quad \Leftrightarrow \\
& w_{3}=w_{2} \bar{w}_{1} w_{0} w_{1} \bar{w}_{0} w_{2}
\end{aligned}
$$

Hence the locally catenative formula

$$
w_{n}=w_{n-1} \bar{w}_{n-2} w_{n-3^{w}} w_{n-2} \bar{w}_{n-3^{w}} w_{n-1} \quad \text {, cut }=3
$$

Notice that the example agrees with the small conjecture.

\section{Example 12:}

This example shows the methods used in example 11 on the DOI-system

$$
H=\langle a, b, c\},\{a-\rangle a b c, b-\rangle a, c-\rangle c\}, a b\rangle
$$

\section{step 0}

$w_{0}^{1}=a b$

$$
\mathrm{w}_{0}^{1}=\mathrm{w}_{0}
$$

$z_{0}=[$ 勋 $]$

\section{$\underline{\text { step } 1}$}

$h\left(w_{0}^{1}\right)=$ abca

$\mathrm{w}_{1}^{1}=\mathrm{ca}$ $w_{1}^{p}=\bar{w}_{0} w_{1}$

$z_{1}=[a b, g a]$

step 2

$h\left(w_{1}^{1}\right)=$ cabc

$\mathrm{w}_{2}^{\prime}=\mathrm{bc}$

$$
w_{2}^{\prime}=\bar{w}_{1} w_{0} \bar{w}_{1} w_{2}
$$

$z_{2}=[\mathrm{ab}, \mathrm{ca}, \mathrm{ba}]$ 
step 3

$h\left(w_{2}^{\prime}\right)=a c$

$\mathrm{w}_{3}^{\prime}=\mathrm{ac}$

$w_{3}^{\prime}=\bar{w}_{2} w_{1} \bar{w}_{2} w_{3}$

$z_{3}=[a b, c a, b c, a c]$

At this point $w_{3}^{\prime}$ has no significant factors, and we have to change some of the previously found generators to obtain significant factors.

This situation occurs exactly in the case where the new generator is a catenation of an initial subword and a final subword of the generators previously found. We mention, without stating the proof, that $[\mathrm{L}(\mathrm{H})]$ in this case is finitely generated. According to the algorithm we now should change the generators to obtain significant factors. But since we know that the group is finitely generated we might as well carry on until we get the locally catenative formula. Notice that although the generators found do not contain significant factors they are still free generators of the group in agreement with the small conjecture.

step 4

$h\left(w_{3}^{1}\right)=a b c c$

$w: 4=\mathrm{cc}$ $w_{4}^{\prime}=\bar{w}_{0} \bar{w}_{3} w^{w} 3^{w_{4}}$

$z_{4}=[a k, c a, b c, a c, c c]$

\section{step 5}

$h\left(w_{4}\right)=\mathrm{cc}$

$w_{5}^{\prime}=\lambda$

$$
\begin{gathered}
w_{5}^{\prime}=\bar{w}_{4} w_{3} \bar{w}_{2} w_{3} w_{0} \\
\bar{w}_{1} \bar{w}_{4} w_{3} \bar{w}_{4} w_{5}
\end{gathered}
$$

From this we get

$$
\mathrm{w}_{5}=\mathrm{w}_{4} \overline{\mathrm{w}}_{3} \mathrm{w}_{4} \mathrm{w}_{1} \overline{\mathrm{w}}_{0} \overline{\mathrm{w}}_{3} \mathrm{w}_{2} \overline{\mathrm{w}}_{3} \mathrm{w}_{4}
$$

And the locally catenative formula

$$
w_{n}=w_{n-1} \bar{w}_{n-2} w_{n-1} w_{n-4} \bar{w}_{n-5^{w}} \bar{w} 2^{w_{n}} n-3^{\bar{w}_{n}} n-2^{w_{n}} n-1
$$

Notice, that although $w_{4}$ is of length 24 we only have to operate on a word $w_{4}^{*}$ of length 2. One can say that we for each word have extracted 
the new information, and the methods used in these examples reduce the hard work involved dealing with fast growing systems.

Acknowledgement.

The main part of the work described in this paper was developed during a workshop on Lindenmayer systems in Aarhus. The authors wish to thank the participants for a most stimulating working atmosphere. We are grateful to Bente Rasmussen for typing the final version. 